\title{
Adénome Pléomorphe Géant à Localisation Submandibulaire: À Propos d'un Cas
}

\author{
Moussa M., \\ Service d'Odonto-stomatologie, Hôpital National de Niamey, Niger \\ Abba Kaka Hy, \\ Service d'Ophtalmologie, Hôpital National de Niamey, Niger
}

Bancolé Pognon Sa,

Service d'Odonto-stomatologie, CNHU HKM Cotonou, Bénin

\section{Camara A.,}

Service de Stomatologie et Chirurgie Maxillo-Faciale,

Hôpital N'Gnace Deen Conakry Guinée

Samuila S.,

Service de Neurochirurgie, Hôpital National de Niamey, Niger

Sani R.,

Service Chirurgie Générale et Viscérale, Hôpital National de Niamey, Niger

Doi: 10.19044/esj.2019.v15n9p185 URL:http://dx.doi.org/10.19044/esj.2019.v15n9p185

\section{Résumé}

Introduction: L'adénome pléomorphe est une tumeur bénigne des glandes salivaires. Ses localisations extra parotidiennes sont rares. L'objectif de ce travail est de décrire la prise en charge particulière de l'adénome pléomorphe dans sa localisation inhabituelle. Observation: Il s'agissait d'un patient de 35 ans, référé de l'Hôpital National de Zinder pour la prise en charge d'une tumeur de la région mandibulaire évoluant depuis 10 ans. Les examens cliniques et paracliniques avaient permis de conclure à un adénome pléomorphe de la glande submandibulaire, par l'imagerie et confirmé par l'examen anatomo-pathologique de la pièce opératoire qui mesurait $15 \times 12 \times$ $8 \mathrm{~cm}$ suite à la submandibulectomie avec résection tumorale complète. Les suites opératoires étaient simples et aucune récidive ni transformation maligne n'a été rapportée après un recul d'un an. Conclusion: La localisation extra-parotidienne de l'adénome pléomorphe est dominée par la glande submandibulaire. Cette localisation se distingue dans ses particularités cliniques et thérapeutiques.

Mots clés: Adénome pléomorphe, glande submandibulaire, Niger 


\title{
Giant Pleomorphic Adenoma with Submandibular Localization: A Case Report
}

\author{
Moussa M., \\ Service d'Odonto-stomatologie, Hôpital National de Niamey, Niger \\ Abba Kaka Hy, \\ Service d'Ophtalmologie, Hôpital National de Niamey, Niger
}

Bancolé Pognon Sa,

Service d'Odonto-stomatologie, CNHU HKM Cotonou, Bénin

Camara A.,

Service de Stomatologie et Chirurgie Maxillo-Faciale,

Hôpital N'Gnace Deen Conakry Guinée

Samuila S.,

Service de Neurochirurgie, Hôpital National de Niamey, Niger

Sani R.,

Service Chirurgie Générale et Viscérale, Hôpital National de Niamey, Niger

\begin{abstract}
Introduction: The pleomorphic adenoma is a benign tumor of the salivary glands. Its extra parotid locations are rare. The aim of this study is to describe the particular management of the pleomorphic adenoma in its unusual location. Observation: This patient was a 35 - year - old patient referred from Zinder Hospital for the management of tumefaction in the mandibular region that had been undergoing surgery for 10 years. The clinical and paraclinical examinations led to the conclusion of a pleomorphic adenoma of the submandibular gland, by imaging and confirmed by the anatomo-pathological examination of the surgical specimen, which measured $15 \times 12 \times 8 \mathrm{~cm}$ following submandibulectomy with complete tumor resection. The follow-up was simple and no recurrence or malignant transformation was reported after a mean follow-up of one year. Conclusion: The extra-parotid localization of the pleomorphic adenoma is dominated by the submandibular gland. This localization stands out in its clinical and therapeutic peculiarities.
\end{abstract}

Keywords: Pleomorphic adenoma, submandibular gland, Niger 


\section{Introduction}

L'adénome pléomorphe ou tumeur mixte est la plus fréquente des tumeurs des glandes salivaires (Gassab et al., 2009 ; Bablani et al. , 2009). La glande parotide est la plus souvent atteinte, les localisations extra parotidiennes sont rares (Queslati et al. , 2003). Les auteurs présentent un cas d'adénome pléomorphe géant extra parotidien évoluant depuis plus de 10 ans sans transformation maligne. Le but de ce travail est de décrire la prise en charge particulière de l'adénome pléomorphe dans sa localisation extraparotidienne.

\section{Observation}

Il s'agissait d'un patient A.Y âgé de 35ans sans antécédent particulier qui a été référé de l'Hôpital National de Zinder pour une volumineuse tumeur submandibulaire gauche évoluant depuis 10 ans. Le patient présentait un bon état général malgré le volume de la tumeur.

A l'examen clinique l'état général était bon malgré le volume de la tumeur. Cette dernière était ferme et fixée au plan profond ; recouverte d'une peau très tendue, sans signe de compression locale ou locorégionale (figure 1). Il n'y avait pas d'adénopathie cervicale palpable. Le bilan radiologique avait objectivé une image bien délimitée, hétérogène avec des logettes multiples.

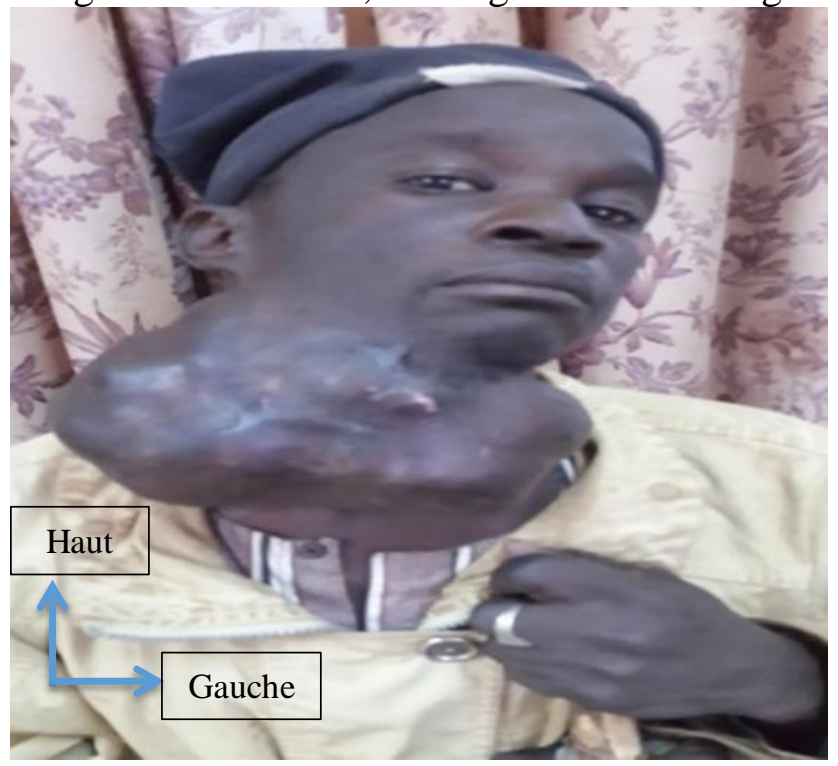

Figure 1: La tumeur en préopératoire

La prise en charge avait été chirurgicale, une submandibulectomie par voie cervicale avait permis l'exérèse d'une masse poly lobulée qui pesait $900 \mathrm{~g}$ (figure 2), le rameau marginal du nerf facial, le nerf hypoglosse et le nerf lingual avaient été respectés. Les suites opératoires étaient simples. A l'examen microscopique, la pièce communiquée montre une prolifération 
tissulaire qui est caractérisée par son polymorphisme épithélial et pseudomésenchymateux. On note une composante myxoide par place périphérique et surtout glandulaire avec métaplasie ; les glandes comportent une couche de cellules myoépithéliales, cubiques et fusiformes. Par ailleurs les cellules myoépithéliales sont plasmocytaires à épithéloides, les noyaux réguliers dépourvus d'atypie, pas de signe de mitoses, pas de signe histologique de malignité évident sur ces échantillons; l'examen a confirmé un adénome pléomorphe. Le reste de l'examen clinique sur le plan stomatologique était normal. Le suivi avec un recul de 1 an (figure 3) était satisfaisant, sans récidive ni transformation maligne.

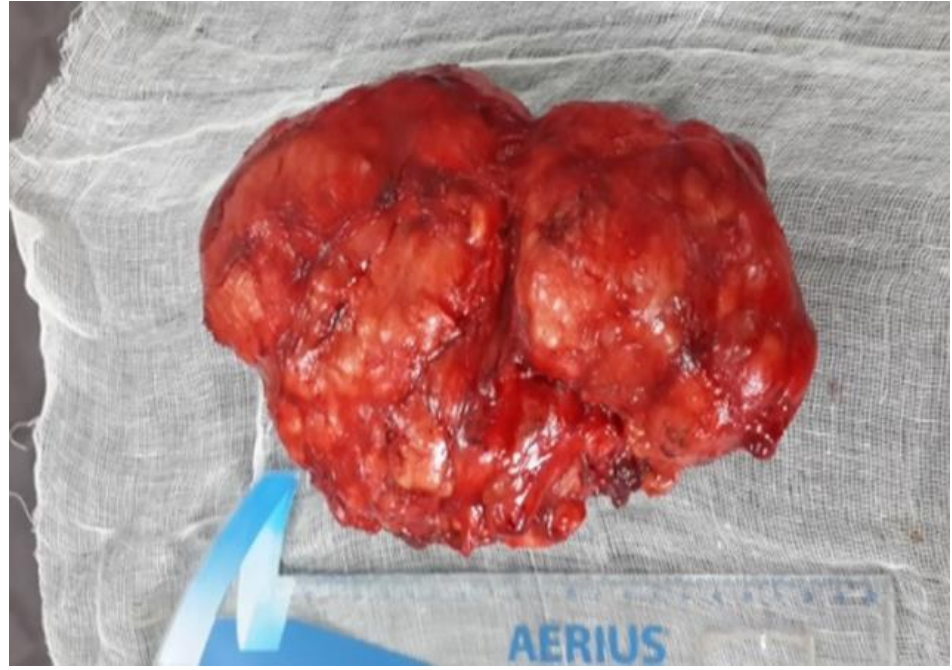

Figure 2: Pièce opératoire : 15 x12 x $8 \mathrm{~cm}$. Poids : $900 \mathrm{~g}$

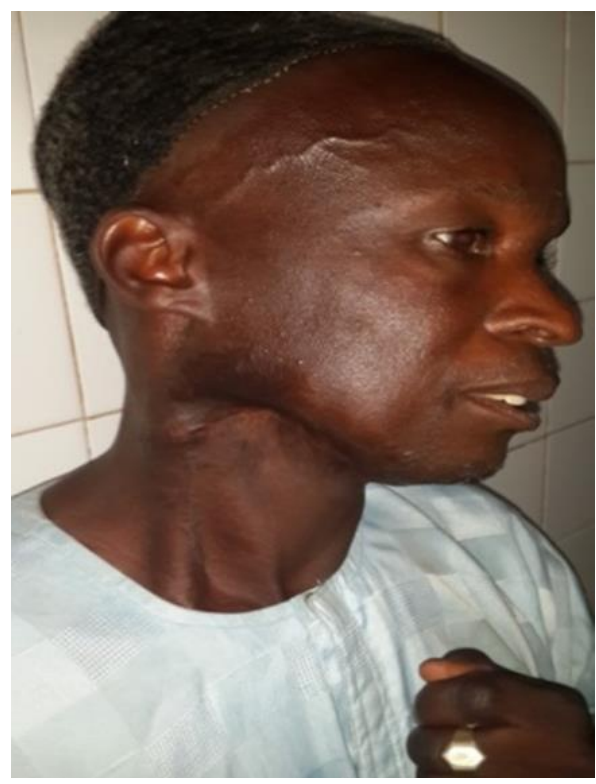

Figure 3: Patient à 1 an post-opératoire avec une cicatrice atrophique 


\section{Discussion}

\section{Au plan épidémiologiques}

L'adénome pléomorphe représente 40 à $70 \%$ de toutes les tumeurs des glandes salivaires(Queslati et al. , 2003 ; Lomeo , 2001 ; Alves et al. , 2002 ; El Kohen et al. , 2007). Il se développe essentiellement au niveau de la glande parotide ; le cas de cette présentation était plutôt aux dépends de la glande submandibulaire. La glande submandibulaire a été reconnue comme le deuxième siège le plus fréquent des adénomes pléomorphes, suivi des glandes salivaires accessoires (Gassab et al. , 2009 ; El Kohen et al. , 2007). D'autres localisations plus rares comme le septum nasal, le vestibule narinaire, le conduit auditif externe, la base de la langue, l'épiglotte et l'espace parapharyngé avaient été rapportées dans des travaux antérieurs (Cwalina et al , 2002 ; Bonfils , 2007 ; Verma , 2002 ; Guerrissi , 2001). C'est la tumeur salivaire la plus fréquente chez l'enfant et l'adolescent. Selon différents auteurs, l'âge moyen de découverte des adénomes pléomorphes varierait entre 36 et 47ans avec une prédominance féminine (Gassab et al. , 2009 ; Lomeo , 2001). Le patient de cette presentation avait 35 ans mais il était plutôt de sexe masculin.

\section{Au plan paraclinique}

L'adénome pléomorphe évolue lentement à bas bruit et est découvert après quelques années d'évolution sous forme d'une tuméfaction isolée de la région intéressée (Ayoub , 2002). L'imagerie est indispensable dans le bilan préopératoire de l'adénome pléomorphe, elle repose essentiellement sur la tomodensitométrie et l'imagerie par résonance magnétique ; cette dernière est l'examen le plus performant pour l'exploration des tumeurs des glandes salivaires et montre classiquement une lésion à limites nettes, avec des contours polylobés et une structure homogène( Lopes et al. , 1999 ; Dubrulle , 2010). L'image obtenue pour ce cas répondait à cette description.

\section{Au plan thérapeutique}

La chirurgie est le traitement de choix pour l'adénome pléomorphe. Selon la glande atteinte et la partie concernée dans cette glande, l'ablation peut être partielle ou totale. Pour les tumeurs de la glande submandibulaire, la submandibulectomie sous anesthésie générale est recommandée. Des submandibulectomies endoscopiques par voie endaurale avaient été rapportées dans des travaux antérieurs (Guerrissi , 2001). Cependant, les voies d'abord conventionnelles restent largement utilisées (Jones , 1987). Quelle que soit la voie d'abord adoptée, l'exérèse de la tumeur doit se faire de manière totale en évitant la rupture capsulaire qui est source de récidive (Bonfils , 2007). Le cas d'adénome présenté dans ce travail avait été traité par une submandibulectomie par voie cutanée sous anesthésie générale. 
La radiothérapie postopératoire, longtemps discutée dans le traitement de l'adénome pléomorphe, est actuellement réservée à de très rares cas récidivants et particuliers. La radiothérapie n’étant pas disponible dans notre contexte, malgré le volume de la tumeur, il n'a pas pu bénéficier de radiations ionisantes.

\section{Au plan de l'évolution}

Dans 5 à $7 \%$ des cas, l'évolution de l'adénome pléomorphe peut se faire vers une dégénérescence carcinomateuse (adénocarcinome ou carcinome épidermoïde). Ce risque de transformation augmente souvent avec la fréquence des récurrences et le délai du diagnostic, il varie de 1,6\% avant 5 ans à 9,4\% après 15 ans. Une récidive locale reste toujours possible même après une exérèse chirurgicale complète (McNamara , 2009 ; Halimi , 2005). Les récidives sont décrites dans $2 \%$ des cas après chirurgie radicale et dans plus de $20 \%$ de s cas après énucléation, elles imposent donc de proscrire formellement toute énucléation ou chirurgie partielle en matière d'adénome pléomorphe (Verma , 2002 ; Guerrissi , 2001). La hantise de la récidive impose une surveillance régulière de tout patient opéré d'adénome pléomorphe (Ayoub , 2002; Jackson et al. , 1993). Le cas objet de cette présentation avait un recul d'un an, et il n'avait pas été noté de récidive.

\section{Conclusion}

L'adénome pléomorphe est la tumeur la plus fréquente des glandes salivaires avec une prédilection pour la glande parotide. Ses localisations extra parotidiennes sont dominées par la glande submandibulaire et les glandes salivaires accessoires du palais et des lèvres. Le cas rapporté dans ce travail était relatif à un adénome pléomorphe géant de la glande submandibulaire. La consultation tardive augmente les risques dans la prise en charge chirurgicale du fait de l'augmentation du volume de la tumeur comme celle rapportée dans cette observation. Les risques de récidive locale et de dégénérescence maligne imposent une surveillance clinique régulière de tout patient opéré d'un adénome pléomorphe.

\section{Conflits d'interet: aucun.}

\section{References:}

1. Gassab E, Berkaoui A, Kedous S. et al. (2009). Adénome pléomorphe à localisation extra parotidienne J. Tun ORL, 22:36-39.

2. Bablani D, Bansal S, Shetty SJ. et al. (2009). Pleomorphic adenoma of cheek : a case report and review .J Oral Maxillofac Surg, 15:39-42.

3. Queslati Z, Naji R, Moukram K. et al. (2003). Adénome pléomorphe de la glande submandibulaire J Tun ORL, 11:19-22. 
4. Lomeo and Finneman J. (2001). Pleomorphic adenoma of the soft palate Otolaryngol head neck Surg , 9:125-132.

5. Alves FA, Perez DE, Lopes MA. et al. (2002). Pleomorphic adenoma of the submandibular gland: clinicopathological and immunohistological features of 60 cases in Brazil Arch otolaryngol head Neck Surg, 128:1400-1403.

6. El Kohen A, Esakali L, Kzadri M. et al. (2007). Adénome pléomorphe de la base de la langue. Rev stomato Chir Maxilofac, 108:215-217.

7. Cwalina P, Skorek A, Narozny W. et al. (2002). Pleomorphic adenoma of minor salivary glands Otolaryngol Pol,56:737-740.

8. Bonfils P. (2007). Tumeurs des glandes salivaires EMC ORL, 20-628B-10

9. Verma K, Kapila K and Kumar N. (2002). Role of fine needle aspiration cytology in diagnosis of pleomorphic adenomas Cytopathology, 13:121-127.

10. Guerrissi JO and Taborda G. (2001).Endoscopic excision of submandibular gland by an intra oral approach. J Craniofac Surg, 12:299-303.

11. Ayoub OM, Bhatia K and Mal RK. (2002). Pleomorphic adenoma of the parotid gland: is long term follow - up needed Auris Nasus larynx, 29(3):283-285.

12. Lopes MA, Kowalski LP, Santos GC. et al. (1999). Clinicopathologic study of 196 intraoral salivary gland tumors. J Oral Pathol Med,28:264-267.

13. Dubrulle F, Martin-Duverneuil N and Moulin G. (2010). Imagerie en ORL, Elsevier-Masson Paris

14. Jones ME and Kaplan MJ. (1987). Surgical therapy of tumors of the salivary glands. In : Thawley SE, Panje WR, editors. Comprehensive management of Head and Neck tumors. Philadelphia : WB Saunders, 1104-38.

15. McNamara ZJ, Batstone M and Farah SC. (2009). Carcinoma ex pleomorphic adenoma in a minor salivary gland of the upper lip. Oral Surg Oral Med Oral Pathol Oral Radiol Endod, 108(5):e51-e53.

16. Halimi P, Gardner M and Petit F. (2005). Les tumeurs des glandes salivaires Cancer/Radiothérapie, 9(4):251-260.

17. Jackson SR, Roland NJ, Clarke RW. Et al. (1993). Recurrent pleomorphic adenoma J.Laryngol Otol,107:546-549. 